\title{
EAl Endorsed Transactions

\section{Use of the cellular automatons (CA) for the discrete simulation of urban sound waves propagation in the smart cities context}

\author{
Eloi Bandia KEITA ${ }^{1}$, Bernard POTTIER ${ }^{2, *}$ \\ ${ }^{1}$ Institute of Technology (IUT), Paris Descartes University and Lab-STICC (laboratory, UBO), France \\ ${ }^{2}$ University of Brest, Brest - (UBO), France - Lab-STICC
}

\begin{abstract}
This paper deals with the problem of sound propagation in urban areas, a real tool leading to smart cities design. It begins by studying the propagation of sound in order to determine and extract the physical parameters that direct this propagation. Then it deals with the formalism of cellular automata and their relation to the specification of physical phenomena with discrete events, such as sound propagation. Then, this technique (cellular automata) is used in a smart city context, to model the propagation of sound, taking into account buildings and other various city obstacles. An implementation is made in CUDA, to simulate this phenomenon on shapefile cards. This work ends with the determination of the sound power in a given location and the geographic area covered by the sound wave.
\end{abstract}

Received on 8 August 2017; accepted on 10 October 2017; published on 20 December 2017

Keywords: Wireless sensor networks, cellular automata, sound propagation simulation, graphics accelerators, parallel programming

Copyright (0 2017 Eloi Bandia KEITA and Bernard POTTIER, licensed to EIA. This is an open access article distributed under the terms of the Creative Commons Attribution license (http://creativecommons.org/licenses/by/3.0/), which permits unlimited use, distribution and reproduction in any medium so long as the original work is properly cited.

doi:10.4108/eai.20-12-2017.153497

\section{Introduction}

Physical world and information systems unification is a major achievement during this last decade. Communication systems, especially wireless technology, and research in physical sensors field have taken a major part in this integration.

The interface between the physical word and a computer can be used in two ways. First, from a local perspective, where such a system can control for instance a single object or provide a personal assistance. Moreover, in a distributed system, this interface collects and analyze sparse data to make decisions, for instance about resource savings : to turn off streetlights, to guide to a parking spot.

Wireless sensor networks are part of this second category. Thus, this thesis is focused on sound

\footnotetext{
${ }^{\star}$ Dr. Eloi KEITA is currently teaching at the Institute of Technology (IUT), Paris Descartes University. He is an associate member of Lab-STICC in the WSN unit of Professor Bernard POTTIER, in Brest.

${ }^{*}$ Corresponding author. Email: eloi.keita@laposte.net
}

propagation simulation in urban environment, in a distributed manner.

The key point of this work is a cellular representation of the city in order to model streets, gardens, ring roads, buildings, and rivers. Geo referenced image analysis, complemented by database consultation, for example to retrieve elevation data, generates this cellular model. Then, this model is converted into a system made of interconnected processes, that can reproduce many collective behaviors, whether physical or digital.

We have developed a cellular automata that models sound propagation, including reflection and refraction, working on a graphics accelerator. Finally, we have produced a coupling method between observation systems by sensor networks and physical systems.

\section{Background}

\subsection{Sound Propagation}

The displacement of a sound wave from one place to another is the transportation of energy, without the transportation of matter. During propagation, at any 
point reached by the wave, the air reproduces the state of the source with a smaller amplitude and a time delay related to the duration of the journey. The delay is a result of the speed of propagation and the distance separating this point from the source. The propagation of the wave implies:

1. that the surrounding environment is the source plastic, a vacuum does not allow propagation of the sound wave,

2. that the source is in a vibrational state: without vibration, there is no generation of sound propagation.

In a compressible medium such as air, the sound propagates under the form of a pressure variation created by the sound source (figure 4). Only the vibration, without displacing the material, is transmitted from point to point between the object that emits the sound and our ear. The sound wave consists of a fundamental and of harmonics that permit the differentiation of different sounds. Sound wave also propagate through solids and liquids, (but not in a vacuum) in the form of the vibrations of atoms called photons. Here also, only the vibration propagates itself and not the atoms which only vibrate around their equilibrium position.

Spatial properties of sound waves. The spatial characteristics of propagation are the following :

- a wave propagates itself, from its source, in all directions possible,

- the disturbance transmits from point to point, according to the current frequency

- the transfer of energy operates without the transport of matter, the waves cross each other without disturbing each other

- the wave propagation speed is a property of the propagation medium.

All sounds (infrasound, sound, ultrasound, hypersounds) propagate identically on the same principle. Several changes take place during the transportation of the sound wave: reflection, diffraction, refraction, interference, absorption and diffusion. Parameters related to the architecture of the city as urban barriers to sound propagation such as: walls, streets, trees, parks, .... will generate modifications that we will detail.

Reflection When a sound wave encounters an obstacle, there is a reflection. The incident wave is reflected and creates a second wave identical to the first, but in a different direction. The phenomenon of reverberation or echo describes an exaggerated and unpleasant persistence of sounds linked to successive reflections bringing the incident wave back to its starting point. The generalized law of reflection reads:

$\sin \left(\theta_{r}\right)-\sin \left(\theta_{i}\right)=\lambda /\left(2 \pi n_{i}\right) \cdot d \phi / d x$, where $\theta_{i}$ represents the angle of incidence to be $\theta_{r}$ represents the angle of reflection We have $d \phi / d x$ representing the phase gradient introduced suddenly at the interface and $n$ the normal vector of the incident plane.

Diffraction When a sound wave is close to an obstacle, it produces a bypass phenomenon. The terminals of the obstacle become sources of secondary waves, which are described as diffracted waves. Intuitively, this is a bypass phenomenon of the obstacle.

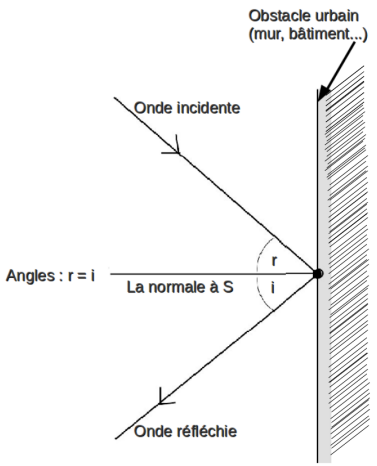

(a) Reflexion of a wave

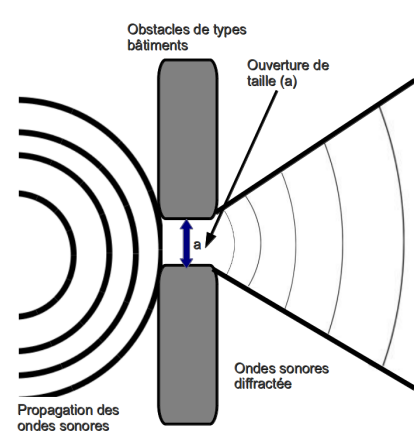

(b) Diffraction
Figure 1. Diffraction - Reflection

Refraction The refractive phenomenon is due to a change to a medium of a different nature. The wave is deflected, its direction modified. One obtains a refracted wave because of the difference in propagation speed of the two medium. This modification also produces a reflected wave. A portion of the energy being absorbed by the obstacle, so these secondary waves are often less intense than the incident wave. The generalized law of refraction is therefore described thus:

$n_{2} \cdot \sin \left(\theta_{2}\right)-n_{1} \cdot \sin \left(\theta_{1}\right)=\lambda /(2 \pi) \cdot d \phi / d x$, où $d \phi / d x$ (meaning that: $\mathrm{d}(\mathrm{phi}) / \mathrm{dx}$ ) represents the gradient of the phase introduced in a sudden fashion to the interface.

Absorption This phenomenon is related in part to the phenomena of successive reflections and refraction of the sound wave. An absorbing medium can be used to mitigate or even eliminate certain sound waves. Similarly a denser propagation medium allows one to obtain a higher speed of sound waves.

Interference By generating multiple identical sound sources located at a certain distance from each other one observes a predictable interference phenomena from the equation of elementary sources. 
Diffusion three types of diffusion are observed when a sound wave propagates depending on the relationship of the wavelength / height and structure of the obstacle:

- back-scattering: here the wavelength is greater than the size of the obstacle (when the wavelength is greater than the obstacle, the sound wave is reflected in multiple directions)

- ante-grade: Here the wavelength is equal to the size of the obstacle,

- multidirectional: here the wavelength is smaller than the size of the obstacle.

This latter multi directional diffusion is that which is the most common and most important during the propagation of sound waves in the city.

These main phenomena related to the propagation of a sound wave usually occur when an obstacle is encountered by the wave. Taking into account the complex architecture of a city, our simulations will simplify the parameters by only considering the walls of buildings, trees and open spaces such as streets or parks. In a city, the propagation of sound waves is constantly deflected because the wave is exchanging with the medium and regularly meets obstacles. Propagation is dependent on the nature of the medium in which the sound wave is propagating.

Elementary metrics : frequencies, wavelength, amplitude, intensity . If we consider a sound wave $\mathrm{Q}$. The equation for its elongation $x$ in relation to time:

$x=A \cdot \sin (\omega t+\varphi)$

A is its maximum elongation, $\omega$ is the pulsation, $(\omega=2 \pi / T=2 \pi f)$ in radian per second and $\varphi$ s the phase shift ( in radian) at the moment t0.

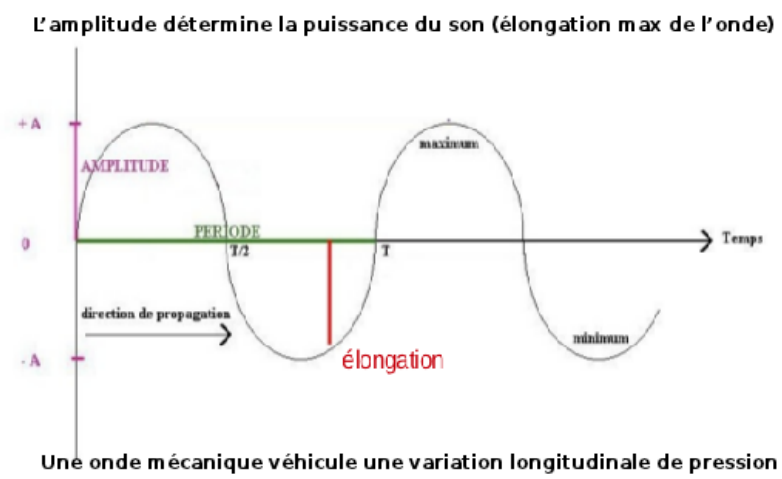

Figure 2. Element of evaluation of a sound wave.

If the sound source vibrates in a sinusoidal manner, on the frequency $\mathrm{f}$, the acoustic pressure, at any point $\mathrm{P}$ of the sound field (the space surrounding the source) is a sinusoidal function of the time of the same frequency $\mathrm{f}$ : $\operatorname{Pac}(t)=\operatorname{psin}(\omega t+\varphi)$ ou $\operatorname{Pac}(t)=\operatorname{Peff} 2 \sin (\omega t+\varphi)$

\subsection{Discrete simulation}

A discrete-event simulation models the operation of a system as a discrete sequence of events in time. Each event occurs at a particular instant in time and marks a change of state in the system. Between consecutive events, no change in the system is assumed to occur; thus the simulation can directly jump in time from one event to the next. This contrasts with continuous simulation in which the simulation continuously tracks the system dynamics over time.Instead of being event-based, this is called an activity-based simulation. Time is broken up into small time slices and the system state is updated according to the set of activities happening in the time slice. Because discrete-event simulations do not have to simulate every time slice, they can typically run much faster than the corresponding continuous simulation. So, in the discrete event simulation, something has to happen so that we can observe and make decisions. It is these moments of modification of the state of the system which is called the event.

\subsection{Cellular Automata Formalism}

Cellular automata (CA) were originally conceived by Ulam and von Neumann in the 1940s to provide a formal framework for investigating the behaviour of complex, spatially distributed systems [1]. Cellular Automata constitute a dynamic, discrete space, discrete time formalism. Space in Cellular Automata is partitioned into discrete volume elements called cells and time progresses in discrete steps. Each cell can be in one of a finite number of states at any given time. The âĂIJphysicsâ $\breve{A} \dot{I}$ of this logical universe is deterministic and local. Deterministic means that once a local physics and an initial state of a Cellular Automaton has been chosen, its future evolution is uniquely determined. Local means that the state of a cell at time $t+1$ is determined only by its own state and the states of neighbouring cells at the previous time t. The operational semantics of a $\mathrm{CA}$ as prescribed in a simulation procedure and implemented in a $\mathrm{CA}$ solver dictates that values are updated synchronously: all new values are calculated simultaneously. The local physics is typically determined by an explicit mapping from all possible local states of a predefined neighbourhood template (e.g., the cells bordering on a cell, including the cell itself), to the state of that cell after the next time-step [2].

\subsection{The Characteristics of Sound Waves}

According to Stanley A Gelfand [3] Sound is defined as a wave that travels through the air in the form of a pressure variation, without material displacement. An example is the vibration of the diaphragm in loudspeakers that transcribes a periodic electrical signal in successive compression and decompression of the air. Sound wave 
also propagates through solids in the form of tiny vibrations of atoms. There are several types of sound, with regard to human hearing and vibration frequency:

- infra-sound : below $20 \mathrm{~Hz}$ one encounters infrasound that we do not hear. Some animals such as elephants, giraffes, whales etc ... may apply the emit and receive up to $10 \mathrm{~Hz}$. Infrasound is a physical reality and can lead to massive destruction at certain frequencies [4].

- sounds of $20 \mathrm{~Hz}$ to $20 \mathrm{KHz}$ are inaudible to humans. According to [5] Blauert, is a sound has a frequency that our ears can sense and interpret, the best sensitivity of human hearing is situated at around $3 \mathrm{kHz}$ with an intensity of about $10 \mathrm{~dB}$.

- ultrasound from $20 \mathrm{KHz}$ up until Megahertz: ultrasound lies beyond $20 \mathrm{KHz}$ and goes up to 1000 $\mathrm{KHz}$. Bats, dolphins and other animals emit and capture ultrasounds of $50 \mathrm{KHz}$ to $150 \mathrm{KHz}$.

- hyper-sound are defined by frequencies beyond several thousand $\mathrm{MHz}$.

Sound frequency ranges

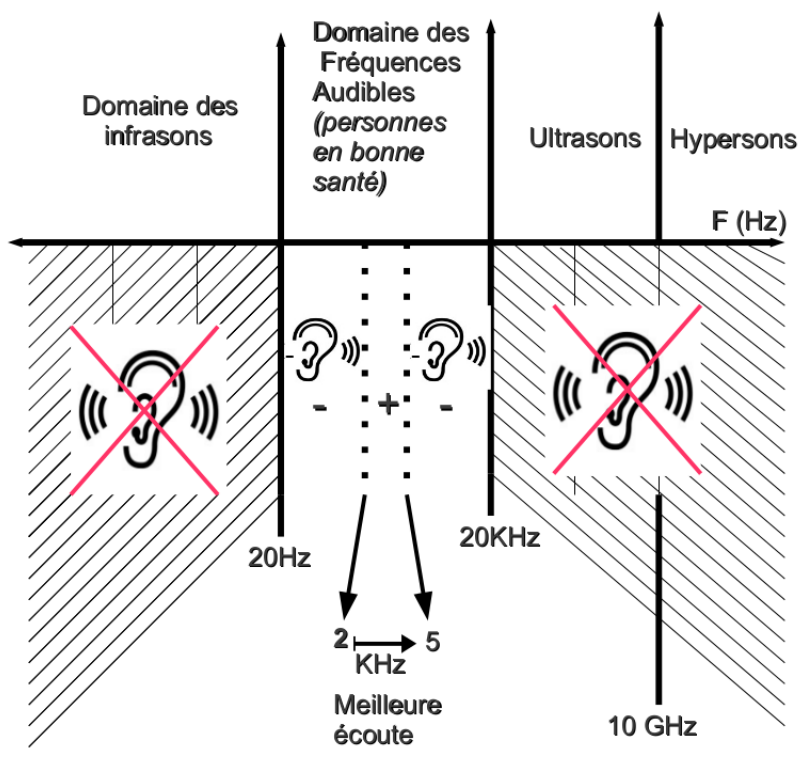

Figure 3. Representation of different types of sound waves.

Definition of the physics of sound. Sound is a longitudinal mechanical wave, a periodic disturbance resulting from the propagation of the vibration of material objects in an elastic medium such as air, water or a solid. It can also be decomposed into elementary sounds that give off wave "noises" that are perfectly periodic, and sinusoidal.

The equation for the elongation of the sound wave as a function of time is
$x=A \sin (\omega t+\varphi)$

A is the maximum elongation, $\omega$ is the pulsation, $(\omega=2 \pi / T=2 \pi f)$ in radian per second and $\varphi$ is the phase shift ( in radian) in the instant $t 0$.

Physics of sound propagation
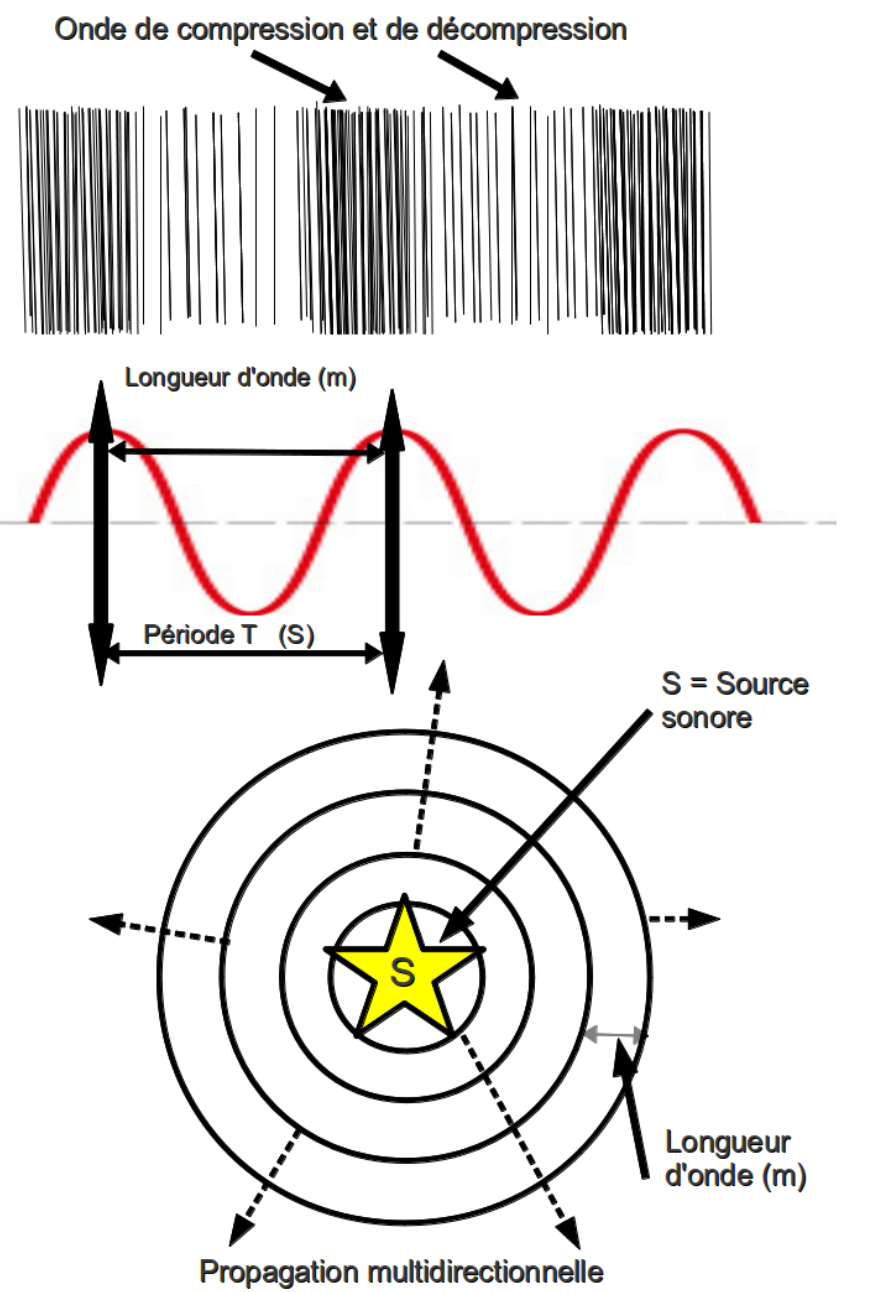

Figure 4. Physical form of pressure waves.

a. The Wave equation and the law of evolution for propagation to one dimension

the partial differential equation (Alembert equation) is given by:

$$
\frac{\partial^{2} u}{\partial x^{2}}-\frac{1}{v^{2}} \frac{\partial^{2} u}{\partial t^{2}}=0 .(1)
$$

For all "f" and "g" functions (assumed regular) there is a solution (with a speed $\mathrm{v}$, but without amortizing or mitigation) which is a function of 2 unknown, "x"(spatial) and "t" (temporal):

$u(x, t)=f(x+v t)+g(x-v t)$.

b. Common parameters of all sound waves These parameters are common to all wave phenomena. They 
are: the wavelength, frequency, speed or velocity of propagation.

The relationships between these three variables are also common: the speed is equal to the wavelength multiplied by its frequency. If one is interested in pure sinusoidal waves, it is possible to qualify several other characteristics: the original spatial direction, the intensity, also called volume or loudness. Other properties related to perception and composition are

tonal pitch and spectral pitch: the pitch of a pure sound corresponds to its vibration frequency (in hertz or $\mathrm{Hz}$, as the number of periodic vibrations per second) according to Claude Abromont and Eugene de Montalembert in "Guide to Music Theory"[6].

In [3] by Stanley A Gelfand and in [5] by Jens Blauert, the authors consider that pitch perception relates to the domain of psycho-acoustic studies and highlight two types of perception of the pitch of a sound:

- the perception of spectral pitch: audible sound oscillates roughly between bass and treble, we can classify all the sounds on a scale;

- the perception of tonal pitch: fairly accurate differentiation of two sounds relatives heard, regardless of their content grading of bass sounds and treble sounds.

Note that the more rapid the vibration of the sound source, the more that the sound will be treble or high. In contrast, the slower this vibration, the sound will be more deep or bass;

\section{the rhythm:}

the rhythm, the sound field is the audible movement of the matter of a sound. The music is dependent on and linked to rhythm but rhythm also exists outside of the music [6]. When there is a succession of sounds and silences of different duration, there is rhythm, while there is not necessarily a melody. By contrary, there can be no melody without rhythm, a continuous sound is not musical. Sound without rhythm is a meaningless wail, but a rhythm and a sound are sufficient to constitute an elementary form of music.

\section{the timbre:}

the timbre of a sound can be defined as the intrinsic colour of the sound, its identity. It varies according to the sound source, irrespective of the first three features. Acoustically and psycho-acoustic the timbre is a very complex concept that depends on the correlation between the fundamental frequency and the harmonics (or partial, according to its relation with the fundamental frequency). The timbre of the human voice is defined as the set of features that help to identify it.[5].

The variation of only one of these parameters produces a perception of different sound. In acoustics, the strength of a sound is measured in decibels, it is a quality linked to a simple correspondence with that which the human ear perceives. This measure is relative to the noise [5], 0 decibel $(0 \mathrm{~dB})$ corresponding to the minimum audible to the human ear. One should note the psycho-acoustic study of theperceived sound intensity is in the light of a given sound physics. These sensations of strong, weak, soft or high sounds are linked to the effective value of the sound pressure.

c. Sound as a spatio-temporal phenomenon There is a direct relationship between space, time and sound: sound travels through space based on a variable time [3]. We recognize three main classes of sounds or acoustic signal:

1. Impulsive: signals that are not repeated in time and have a fixed envelope.

2. Periodic signals in which the form is repeated through time.

3. : Aleatory : signals which are not periodic..

We will pay particular attention to the class of impulsive sound signals in another part of this study.

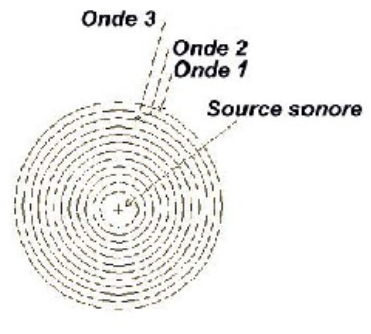

(a) Sound wave train

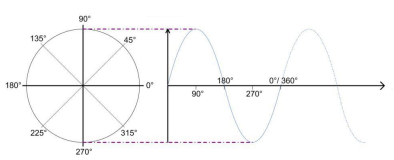

(b) Wave length
Figure 5. Trigonometric representation of physical sound

\section{3. related work}

In [2], authors discuss the formalism of cellular automata and their relation to the specification of discrete event systems. In this work, both the Cellular Automata(CA) and the DEVS(Discrete Event system Specification) and parallel DEVS formalisms are introduced. Then, a mapping between Cellular Automata and parallel DEVS is elaborated. This fills in the CA -> DEVS edge in the FTG(Formalism Transformation Graph). The mapping describes CA semantics in terms of parallel DEVS semantics. As such, it is a specification for automated transformation of CA models into parallel DEVS models, 
thus enabling efficient, parallel simulation as well as meaningful coupling with models in other formalisms.

Authors of [7] investigate several aspects for efficiently implementing a sound propagation processor as a nonlinear cellular network. Starting from a partial differential wave propagation equation, they define an equivalent cellular array to simulate a certain 2dimensional scenario space where various obstacles and signal sources may be positioned arbitrarily. The defining equations in the initial model with 5 state variables per cell are redefined into a much faster model with 2 state variables per cell.

In [8], authors deal with the sound event detection in a noisy environment and present a first classification approach. Detection is the first step of their sound analysis system and is necessary to extract the significant sounds before initiating the classification step. They present three original event detection algorithms. Among these algorithms, one is based on the wavelet and gives the best performances. They evaluate and compare their performance in a noisy environment with the state of the art algorithms in the field. Then, they present a statistical study to obtain the acoustical parameters necessary for the training and, the sound classification results. The detection algorithms and sound classification are applied to medical telemonitoring.

\section{Towards an interweaving of urban sounds}

The first part of this paper presents partitioning techniques and a generation of cellular systems. These techniques can be applied to the urban environment and apprehended through electronic maps or aerial photos, or more directly by querying geographic information systems. These cell systems will represent elements such as streets, buildings, gardens, waterways, public or commercial facilities: schools, stadiums, shops and supermarkets ... The capture of physical information is also on the agenda although its mapping is less publicly present. The equipment related tosmart cities its development in recent years is: electronic parking management by wireless sensor networks, but also detection of air pollution or detection and control of night lighting .... The purpose of this paper is a proposal for modelling and simulation of the propagation of sound waves in urban areas and its implementation on parallel architecture. This proposal is of course based on the concepts outlined in the 3rd chapter of my thesis . ${ }^{1}$ Mapping sound is obviously more difficult than the analysis of more static data. Sound is essentially a transient phenomenon linked to a

\footnotetext{
${ }^{1}$ http://faqbr.com/pub/modeles_physiques_et_perception_analyse_du_milieu_sonore_urbain_pdf.html
}

physical cause, of which the duration may be brief (the passage of a motorbike), very short (a cry), recurrent (recreation in a school yard) or quasi-steady (road). The simulation of the sound can be used as a prediction for noise pollution and for the coupling of diagnostic or monitoring networks. Some of these are already classified. The National GIS propose thus some segments documentation of noise nuisance in proximity to circulation routes [9]. Figure 6 presents two systems of cellular sounds calculated using official GIS Cartelie [9] in the proximity of the University of Brest.

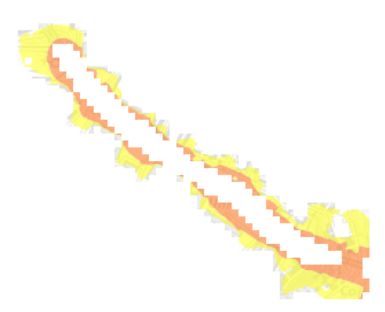

(a) zone 1

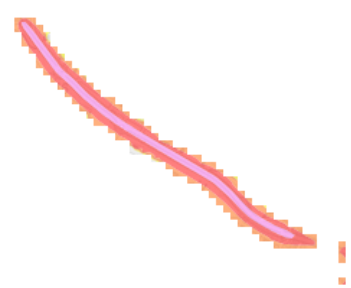

(b) zone 2
Figure 6. Two joint sound cellular systems extracted from public mapping. One can recognize at the centre of zone1 a road intersection, at the extreme right side, a bridge which diffuses the pollution on a larger scale..

\subsection{Classification of propagation elements}

Sound mapping can therefore serve to improve wellbeing by permitting the reduction or isolation of pollution/nuisances. This mapping could also enable the follow up of exceptional/unusual or regular events and to intervene and advise regarding digital augmentation for the hard of hearing.

For the analysis of noise, we can build on zones with differentiated features that we configure in cellular systems, for example:

- obstacle free zones where the sound propagates freely,

- wooden or vegetative zones that are able to reduce sound propagation

- walls of buildings with the capacity to block propagation or otherwise to perform a propagation with particular physical characteristics or a reflection,

- specific zones of activities, noise generators.

For a given cellular system, for harnessing a propagation, the first task will be to determine the characteristics of the diffusion of a wave emitted by a source. A more complex situation is obtained by the composition of the different cellular systems that will 
filter the sound by transforming it. These compositions derive from representations of urban structures or three dimensional topography in the zones of interest.

This chapter will present results concerning the cellular modelling of sound and its simulation with regard to simplified features formed in urban areas conductrices (open air) and obstacles (walls).

The bibliography concerning the cellular sound approach will also be used to explain how to simulate the propagation of sounds according to the parameters applicable to complex systems. From the simulation results, we can make several estimates. One can in this instance decide on:

- The zones of perception : These zones correspond to surfaces on which a sound is audible from the sound source. These are areas such as car parks or sports grounds;

- disturbances: these are disturbances related to the propagation of sound waves such as buildings, walls, metal structures;

- parasitic propagation : here we envisage the detection and location of undesirable sound waves that are able to be superimposed on a sound. This detection can be done at the level of frequency differences, of intensity and location of the sound source;

- reflections, refraction, diffusion : These are phenomena related to the propagation of sound waves in an environment not free of obstacles, which will be calculated here on the basis of the barriers to the propagation, typically buildings.

\subsection{Quantitative application Motivation}

The upstream result of the specific deployment of physical sensors, several benefits are expected from this evaluation :

- maps of noise pollution (for example usable for urban planning) : noise pollution/nuisance is an important social and health threat. This nuisance is often the consequence of human activities such as excessive urbanization, transport (figure6) industrial activity. By intervening in the planning of urbanization, it is possible to improve the impact of noise pollution in a significant manner ;

- alerts regarding critical events: it could be repeated shouts, noise from accidents or explosions... It is then necessary to construct a means of automatic alert, integrating the location of the source and signalling to it the competent authorities. Regarding extreme measures, it is also necessary to think of the disaster areas and the need to find victims using sound signals;
- statistical observation: involves obtaining quantitative and eventually qualitative information, the physical sound itself being information that enables porter other information from a higher level. It is then captured and / or stored, possibly to convey information based on the use or need envisaged, in accordance with legal provisions.

Noise has always been a significant physical signal, the Indian "listened" to the vibration of the rails or gallops. Automatic perception enables similar diagnostics, perhaps at a level that is superior to vision. The flow of automobile traffic thus has certainly more sound signatures characteristics in the absence of vehicle, of normal flows or congestion.

\subsection{Social signification of sound}

The sound is a natural environmental phenomenon, a richly composed, multifaceted world. Sounds tells the story of the city by drawing our way of living by other means than pictures or writing. It often signals the presence of life and many other things in space and time. This is data that can be interpreted in conjunction with other information, such as geographical, for example. Sound can be considered as a critical element in an information system. One can list a few pointers here: traffic, passengers in public transport, public works machinery, factories, the tiny beating heart heard by the obstetrician, the ambulance sirens, children in a recreation yard, a square, a summer camp, passing aircraft, the domestic life of the inhabitants, markets, events, parties, mass movements, wild animals in the forests, birds, thunder, wind, ringtones, footsteps in the corridor, the rhythmic variations of urban transport, sudden braking on the asphalt ...

A social environment sometimes bears startling acoustic contrasts depending on the moment, of the period or the place. These contrasts reflect difference organizational order and are indicative of a social environment. In making a geographical correlation in areas of interest with historic sounds, can lead one to observation of the sound mapping of this area and its identification. This map is a witness of a human geography, social behaviour, a living environment. We deal in this chapter with a new way of appropriating our environment through its sound identity. We will look at different aspects of city noise and the interactions with these sounds:

sound capture for listening, for a virtual walk, based on the study of the propagation of urban or even rural or local sound waves.

Research new techniques for capturing and analyzing the sound identity of our environment, an environment with countless phonic signatures. 
Temporal and spatial location in the situation of the overall sound environment with the ability to stop on a detail, to amplify, affirm or refine a sound signature : sounds of natural elements, foreign languages in transitory or commercial environments.

Reality at any given time is thus interpreted to emphasize a particular event, an indication, a signal, an identity, etc. A social environment without noise would be to follow a televised musical concert with it sound cut. To be able to convey meaningful sounds in the city is an important element in the information society of the future.

\subsection{Physical propagation simulation approach}

We have chosen a cellular model proposed by Radu and Ioana Dogaru in [7]. The proximity to the cellular automaton being used is a Von Neumann radius 1 with neighbourhood cells consisting of North, South, East and West. The variables of state of the cells include the presence of buildings, one or more source (s) sound (s), physical parameters specifying a sound. The transition function operates on the parameters of mitigation and diffusion of the sound wave, by reconstructing the local developments thereof. A practical realization has been constructed, including cellular systems integrating data from an image extracted from navigation on OpenStreetMap in the city of Brest. Each pixel is associated with a zone of a few meters wide depending on the scale of the map, eg $45 \mathrm{~m}$ wide for each pixel for the first simulation shown Figure 9. It has a map of the buildings in the city provided in the form of shapefile, file format, nearly 80,000 polygons (buildings) represent thus potential obstacles for sound. The simulation allows exploration fictive interactive through a virtual microphone that reproduces the sound simulated after propagation, and all this in arbitrary locations. Before going into the details of the simulation, however, it is necessary to look at the physical nature of sound and its discrete data abstraction.

\section{Simulation of sound propagation by cellular automata}

Cellular automata constitute a way to model complex systems such as the phenomenon of wave propagation, diffusion or gas flow. The reason is that the spatial influences are calculated locally like the real physical phenomenon. In a model of propagation simulation by cellular automata, we consider a spatial grid of cells that evolve according to their state and the state of their immediate neighbours. A complete cellular model is a scenario in which the spatial dimension integrates:

- the existence of cardinal directions and positions reachable from the cells; the neighbourhood used for the sound modelling is a Von Neumann of radius 1.

- Changes in the state including the context, barriers, walls, trees as well as noise sources.

Regarding the discrete space, it will be necessary to give each cell a transition function to enable the description of its changes and evolution.

The space is therefore represented by a planar array of cells. This grid could, however, be constructed in three dimensions. Each cell can, at any given time, be in a finite number of states. The update rules are the same for all cells. Each time the rules are applied to the entire network a new generation is produced.

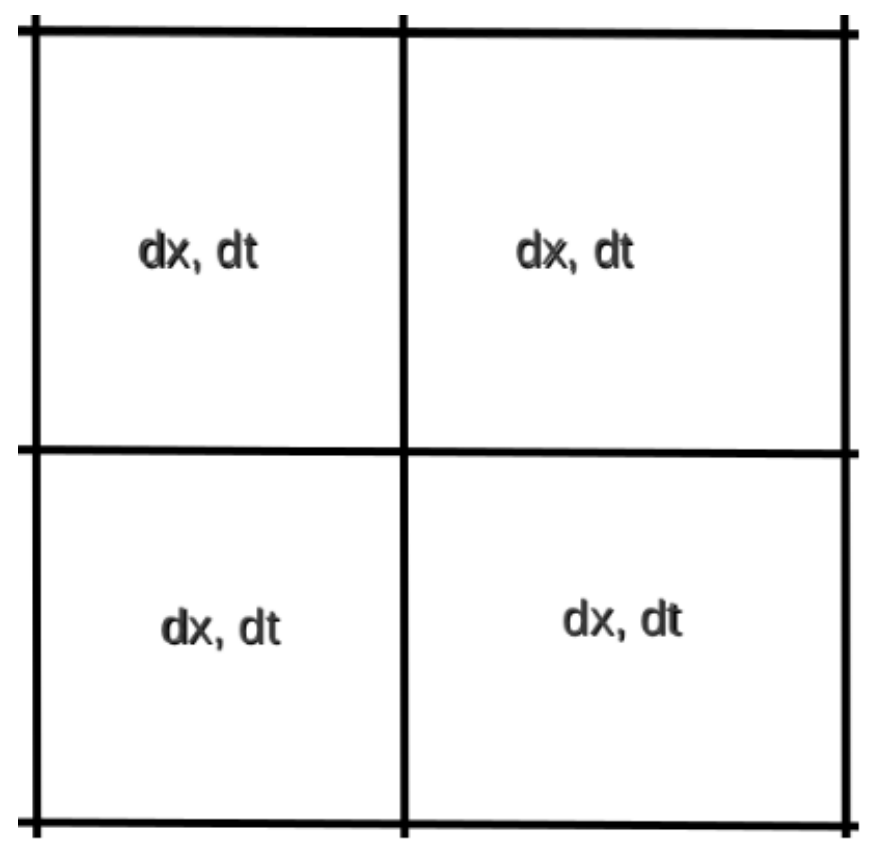

Figure 7. Cell Matrices.

\subsection{Transition function: neighbourhood, pressure and speed}

Conforming to the proposition put forward by Radu and Ioana Dogaru in [7], we are assuming partial differential equations 8 representing the equations of sound waves, then we define the discrete sound propagation model.

\subsection{Interpretation of sound diffusion}

This interpretation is the target of a simulator which generates sound waves in an urban environment. These waves travel from place to place, are reflected or amortized. The findings of these changes are the subject of the simulation, for diagnosing the power of the sound, for example.

Several levels of explanation can be distinguished with respect to the simulation: 


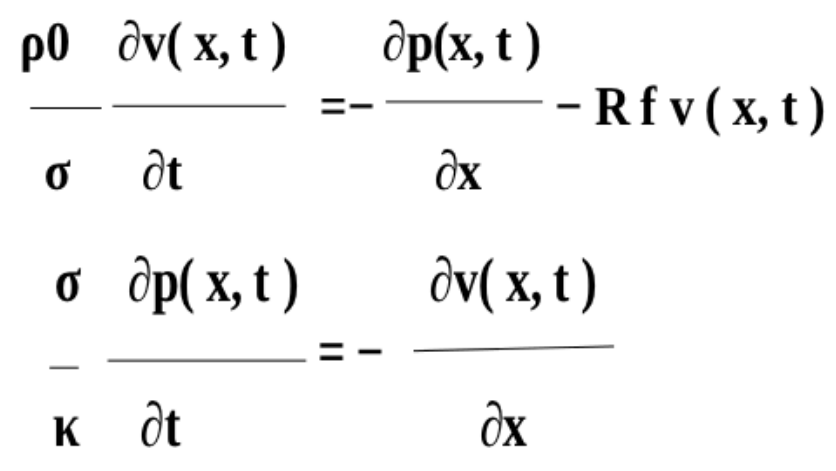

Figure 8. Partial differential equation (the Alembert formula).

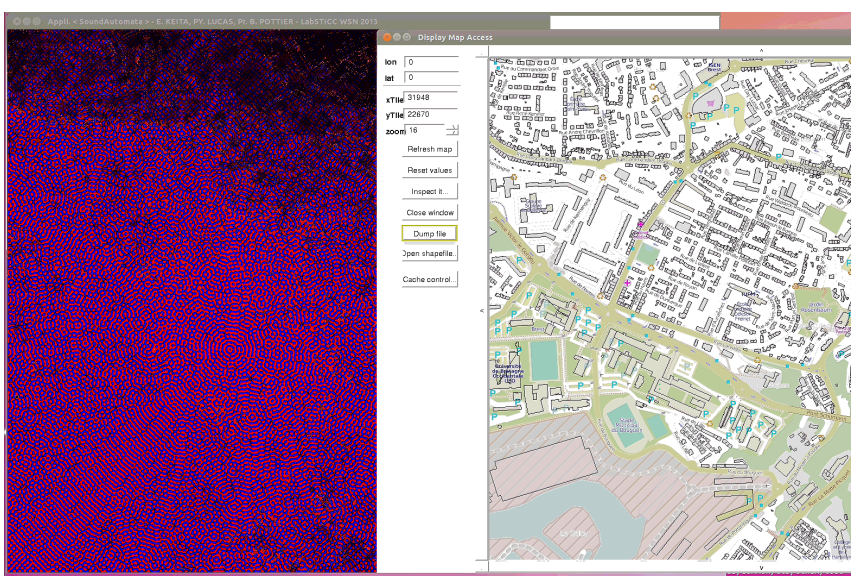

Figure 9. Propagation simulation in Brest: the map of the buildings is shown on the right, while the mottling to the left represents the pressure pockets of a sound source. This landmark has its concentric circles south of the centre of the cellular automata.

1. the parallel, abstract model, which is that of the cellular automata,

2. the sequential execution of this model where we pass from one plane at the moment $t$ to the following plane at the moment $t+1$, with second plane being produced by scanning rows and columns,

3. parallel execution where transformations are performed on a parallel machine.

It is of course interesting to observe the relative performances of the two executions, given the ambition in terms of the computational load. We have used various studies from the two procedures.

\section{Sequential implementation and validation}

\subsection{State of a cell and transition function}

The parameters of the state. Each cell is updated at each discrete time step according to a rule of local interaction. First, the particle velocities in the four directions are updated as a function of time, in accordance with the pressure difference between neighbouring cells. The rule for updating is given by: [10]

$V^{\prime} a(x, t+1)=V a(x, t)-P(x+d x a, t)-P(x, t)$.

Is going to represent the particle velocity and $P$ the sound pressure. The position of the cells is expressed as a vector $(x)$ in steps of discrete time (t). The suffix (a) in dxa represents the index of the four neighbours. The particle velocity obeys also:

$V a(x, t+1)=(1-d) \cdot V a(x, t+1)$

This formula expresses the linear energy dissipation mechanism [10]. This state integrates the sound pressure P. The update of this pressure during the cellular automaton stages is written:

$P(x, t+1)=P(x, t)-\mathrm{C}_{a}^{2} \sum_{a} V a(x, t+1)$

With $C a=$ the speed of waves traveling in the $A C$ area , Va represents the particle velocity and $P$ is the sound pressure.

The absorption coefficient (or constant amortizing of the sound in the obstacle) is another parameter of the cell. In the case of air, one uses :

$\mathrm{d}=0.0001$ This coefficient can reach up to 0.8 for current urban obstacles.

In CFL conditions (Current-Friedrichs-Lewy or Richard Current's Number), dimensionless numbers - according to Toshihiko Komatsuzaki in "Modelling of Incident Sound Wave Propagation Around Sound Barriers Using Cellular Automata" we have:

in the case of a one dimensional pattern: $C o=\frac{v \Delta t}{\Delta x}$ with : $v$ (speed in the direction " $x "$ ), deltat (time interval) and deltax (dimensional interval).

for dimensional patterns ( $\mathrm{n}$, here 2 ), the equation is written as :

$C o=\Delta t \sum_{i=1}^{n} \frac{v_{x_{i}}}{\Delta x_{i}}$.

The values of each dimensional interval can be chosen independently of each other.

The speed of sound in air under normal circumstances at 20 degrees Celsius: $c=344 \mathrm{~m} / \mathrm{s}$

For the cellular automaton, under CFL conditions the maximum wave velocity becomes: $C a=1 /$ ấŽ̌2. We can therefore take a speed, for reasons of applicability, inferior to $1 / \hat{a} \mathbf{L} \check{Z} 2$

$\mathrm{Vca}=0,688-(\mathrm{so}, \mathrm{dx} / \mathrm{dt}=500)$

With $V c a=$ the selected speed of the cellular automata .

With this data, we propose that the code can be written as follows :

void step (int i, int j)

$\{$ double p, v0, v1, v2, v3 ; 
Comparison :physical system - AC Model

\begin{tabular}{|l||l|l||l|}
\hline Systems & $\begin{array}{l}\text { Speed of } \\
\text { sound }\end{array}$ & steps of time & Size Cells \\
\hline Physical & $\begin{array}{l}\mathrm{c}=344 \\
{[\mathrm{~m} / \mathrm{sec}]}\end{array}$ & $\begin{array}{l}\mathrm{dt}=1 / 344 \\
{[\mathrm{sec}]}\end{array}$ & $\begin{array}{l}\mathrm{dx}=0.001 \\
{[\mathrm{~m}]}\end{array}$ \\
\hline AC 2-dim & $\begin{array}{l}c<1 / \hat{\mathrm{L} L} \mathrm{Ž2} \\
{[\text { cell/step }]}\end{array}$ & $\mathrm{dt}=1[\mathrm{step}]$ & $\mathrm{dx}=1$ [cell] \\
\hline
\end{tabular}

Table 1. Parameter Equivalence: comparison of the parameters defined in the automaton with those of the physical system [10].

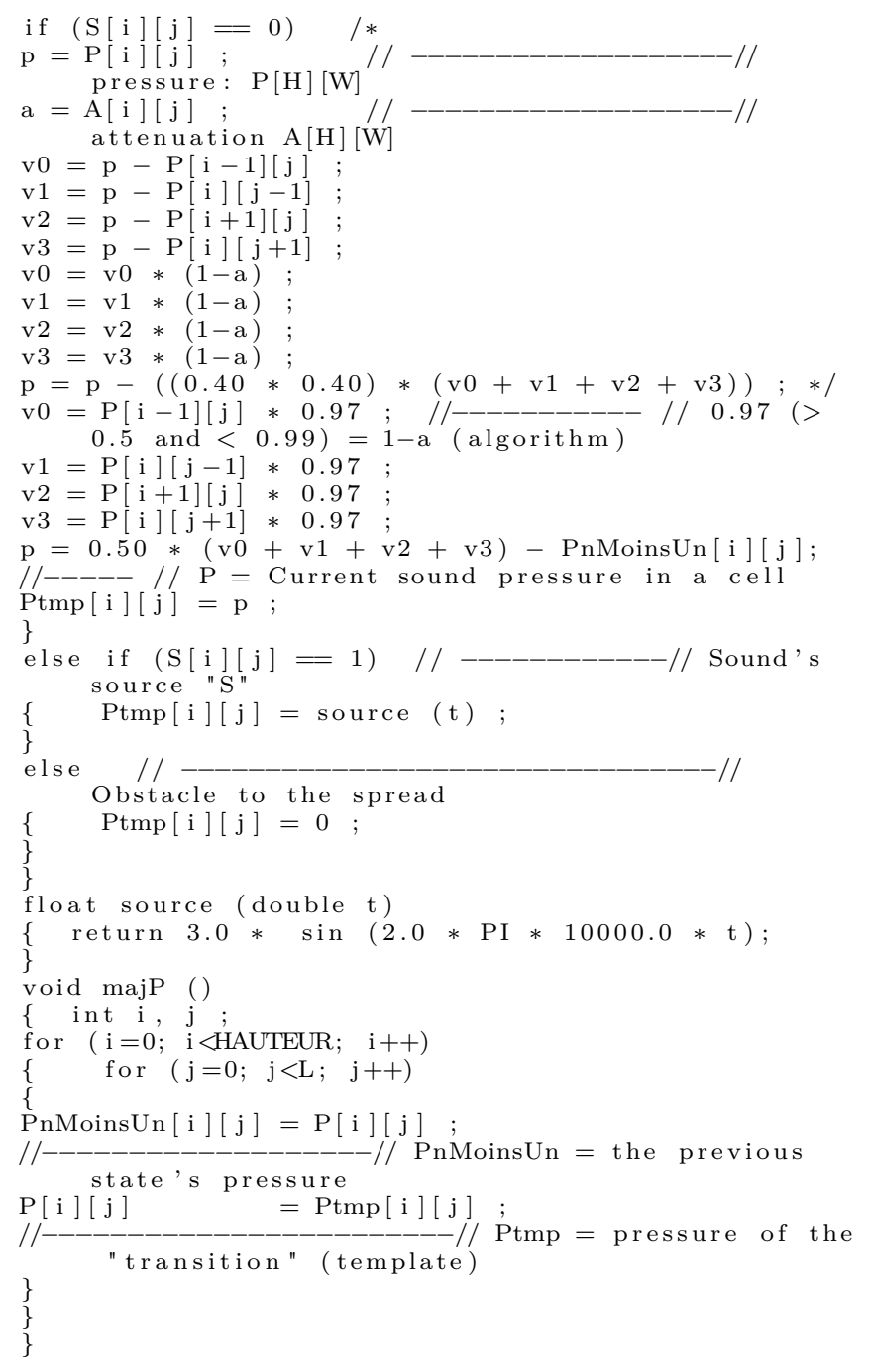

\subsection{The cell modelled in the environment}

An average particle size for this simulation is a grid of 1000 X 1000 cells representing one million elements. To obtain a clear idea, we can assume a wave frequency to $10 \mathrm{KHz}$, corresponding to a period of 100 microseconds. The sound propagation speed is $344 \mathrm{~m} / \mathrm{s}$ in an atmosphere under normal pressure conditions, and temperature to 20 degree Celsius. The space occupied by a sound pulse is the wavelength of the value of $3.44 \mathrm{~cm}$. By rheasuring a cellular automata to $10,000 \mathrm{~Hz}$, a grid whose grain is $3.44 \mathrm{~cm}$ would cross a cell of this size in each period. In Figure 10, the propagation is observed from 3 sound sources composed of interference, diffraction and refraction.

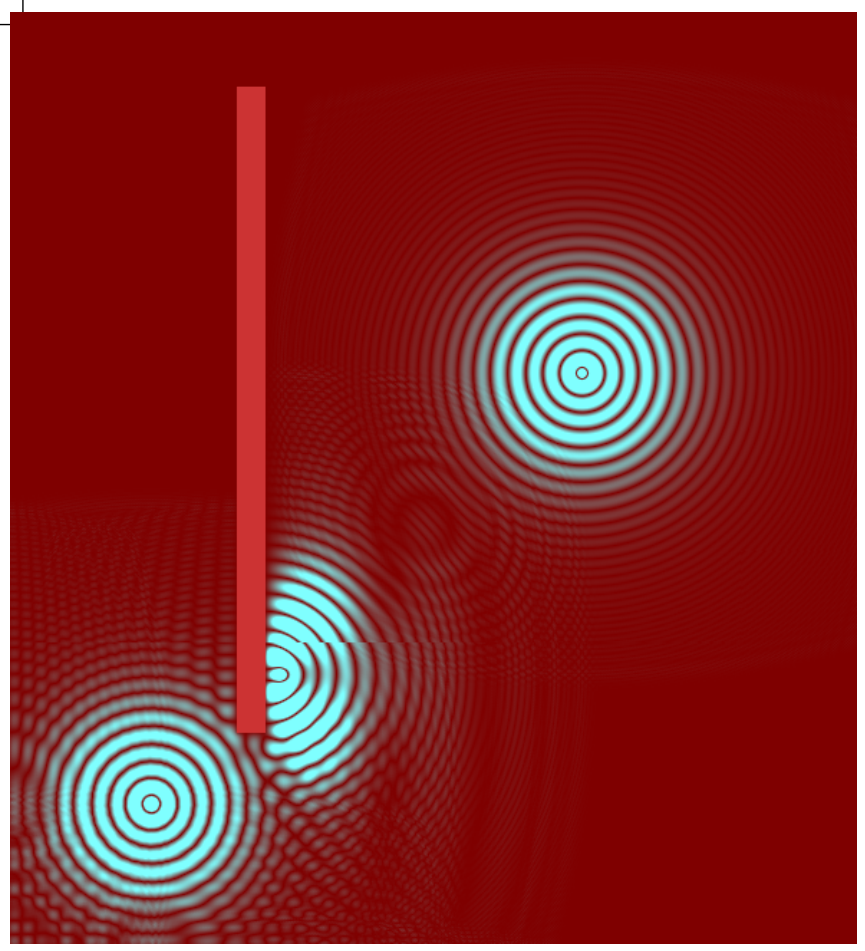

Figure 10. Simulation of propagation of 3 sound sources, with detectable wavelengths (blue stripes) and a red bar symbolizing an obstacle.

The vertical bar illustrates a wall with a coefficient mitigation greater than 0.8 (resistance of the walls). It permits the observation of the phenomena :

- of the interference when the waves trains from the 2 sources meet,

- of the reflection during the contact of the waves

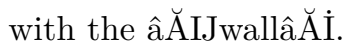

The period: $\mathrm{T}($ period $)=1 / \mathrm{f}=0,0001 \mathrm{sec}$. The wavelength $\lambda$ is written as: $\lambda=V T=\frac{V}{f}$ This wavelength is observable in the figure: it corresponds to the distance between two blue circles. The pulsation or angular frequency is given by: $\omega=2 \pi f=$ $2 \pi / T$

The sound pressure at a point thus calculable.

\section{Spatial aspects and parallel execution}

It is interesting to represent parallel behaviour by the parallel execution structures. Sequentially encoded in a first step, the cellular automata has therefore been 
transcribed for a NVIDIA graphics accelerator and programmed in CUDA. At the same time, the structural data for the city has been translated to enable the cellular system to run.

\subsection{Geometry of the city and sound waves}

Here, the physical dimension becomes important, because cellular systems represent real physical objects whose geometrical dimensions are known. Depending on the scale of the overall geographical position of the area of interest or city, we observe different AC cells sizes whose granularity can also be varied. In Figure 11, the Geo-localization data presented on the top left of the interface conducts a coordinate of 1.586 metre per pixel. Urban structures will behave both as filters and reverberation systems.

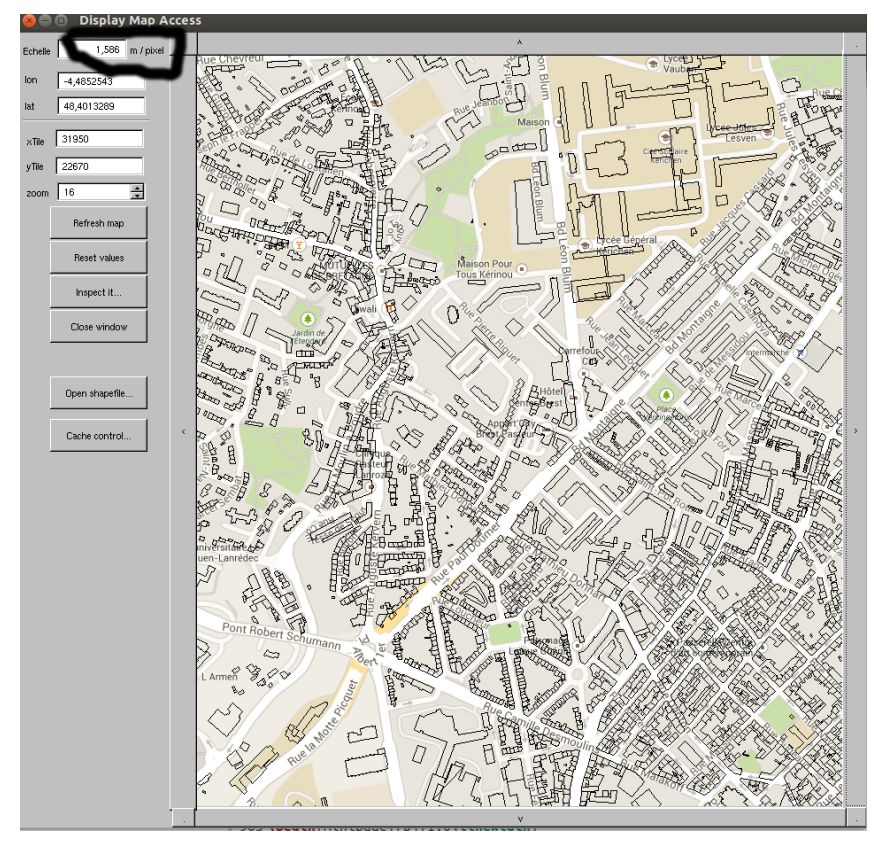

Figure 11. Calculating the size of the cell with the integration of a Geo-localized map of Brest. (The tool is one of the projectors developed by Pierre-Yves Lucas.)

The instantaneous acoustic pressure is an integration of pressure over a period $t_{i}$,

it oscillates around the ambient pressure $P_{a}$.

We consider variations around a stable pressure : $p^{\prime}\left(t_{0}\right)=p\left(t_{0}\right)-P_{a} .=p\left(t_{0}\right)-\int_{t=0}^{\infty} e^{-\frac{t}{\tau}} p\left(t_{0}-t\right) \mathrm{d} t$ $\tau$ to permit the determination of sound pressure frequencies.

The effects of sound on the ear depend on the power of the sound waves which determines the volume level. The sound power is proportional to the square of the acoustic pressure.
The effective value $p_{\text {eff }}(t)$ of the acoustic pressure is calculated over an integration period $t_{i}$ :

$$
p_{e f f}(t)=\sqrt{\frac{1}{t_{i}} \cdot \int_{t-t_{i}}^{t} p^{\prime 2}(t) \mathrm{d} t}
$$

The Sound Level Meters allow the inclusion of the human perception of different sounds to its real sound level by performing a weighing of the results of noise measurements, by frequency [3].

Figure 12 shows the relations between the pressure and the neighbourhood of a cell.

\subsection{Representation of the environment}

Here, a preliminary scale calculation using data from the Mercator tile projector adapts the scale of the map of a city to the size of AC cells. The meter pixel conversion formula is integrated with laboratory tools to directly give the real environmental space measured in meters per pixel.

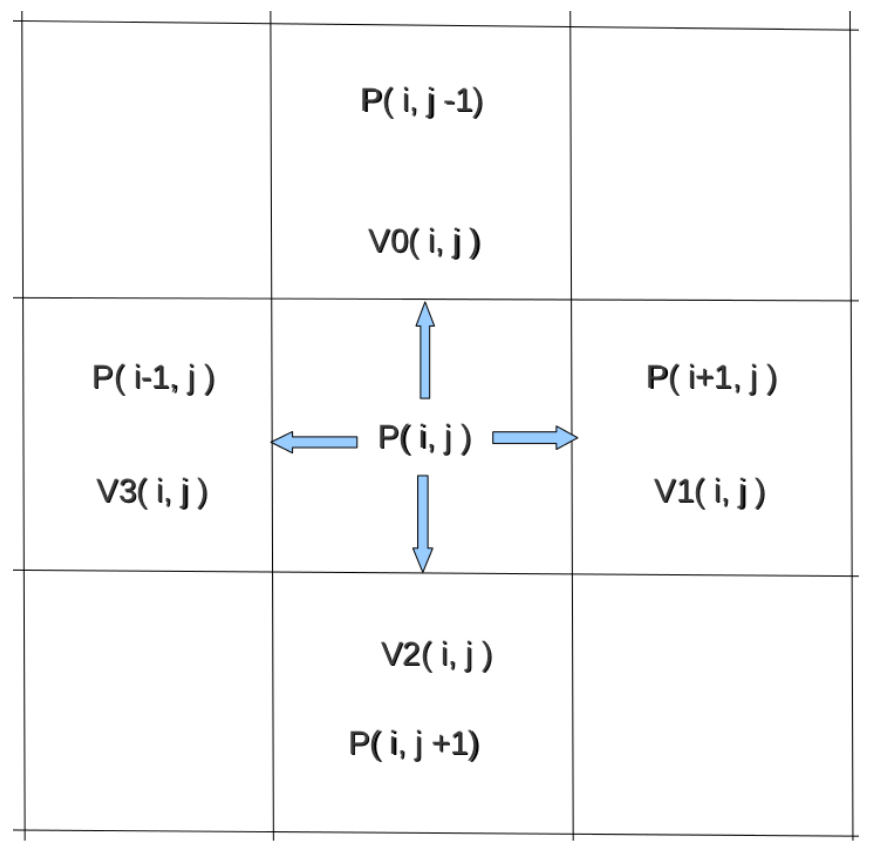

Figure 12. Pressure and speed in each cell

\subsection{Execution parallel on CUDA}

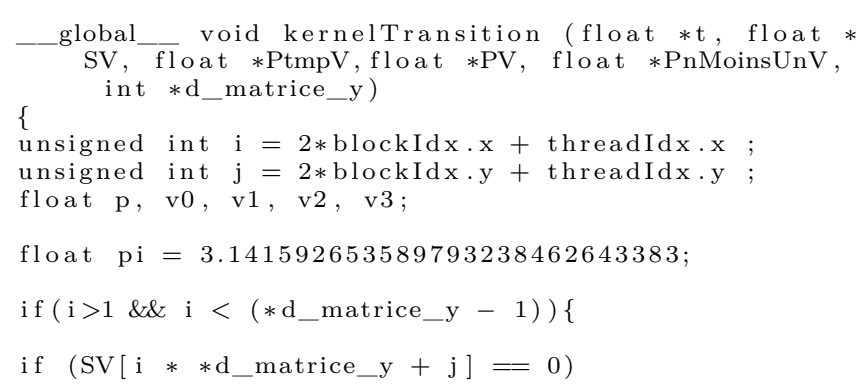




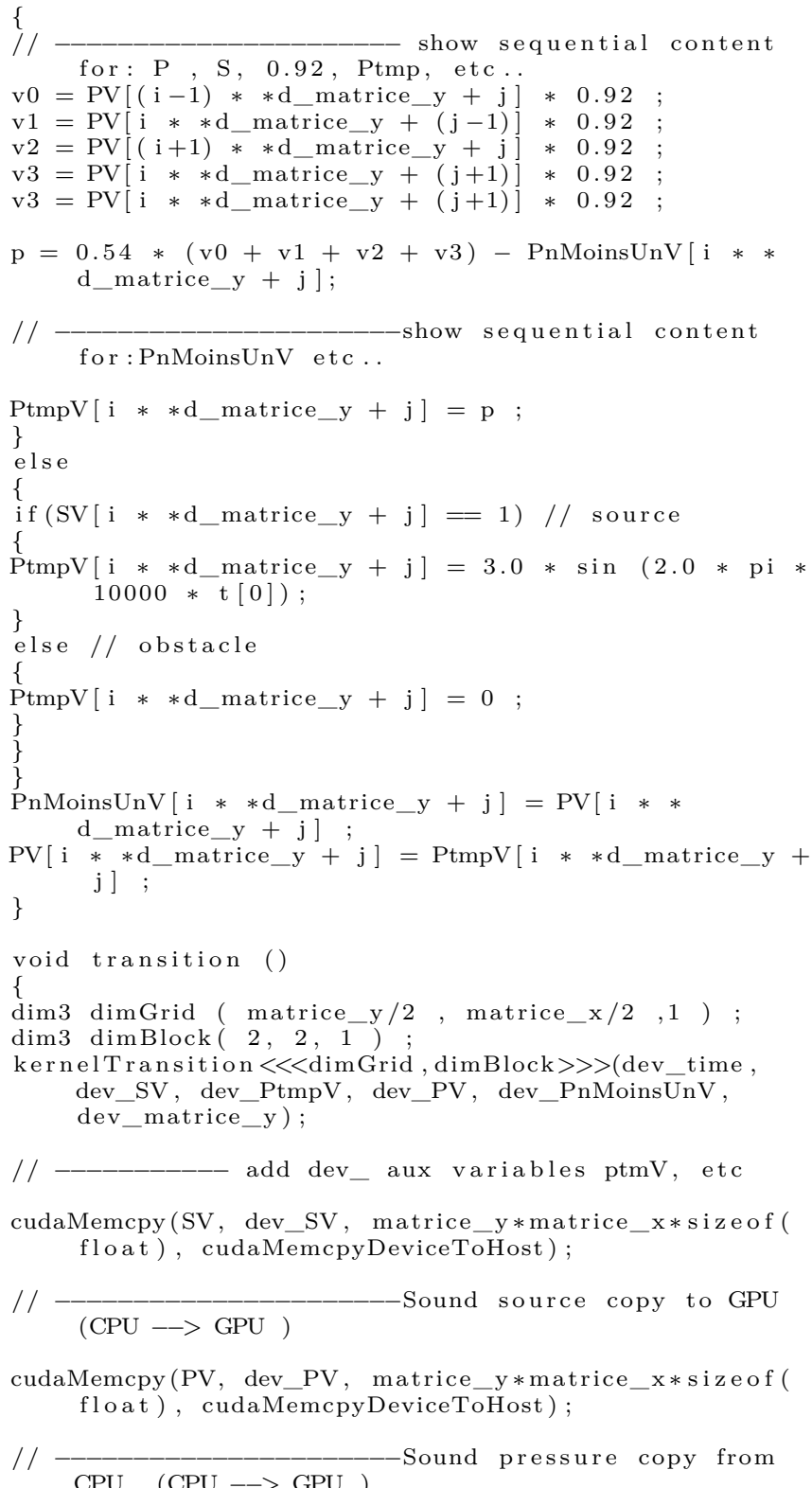

Manual determination of the parameters of interactions and visualization of a sound in a cell. It always considers two state variables placed in each cell $\mathrm{Cf}$ Figure: 12 the sound pressure " $\mathrm{P} "$ and the speed of particles "V".

\subsection{Statistical analysis of cellular plan}

After recovery of the cellular plan, it is possible to make some statistics or to represent the sound characteristics as volumes. Figure 15 shows the distribution of the sound pressure over the map of the simulated site. We can recognize the source and areas susceptible to this pressure, or on the contrary, protected areas.

\subsection{Virtual microphones}

This plan can be produced, analyzed and displayed. Preliminary data concerning the coverage of a sound

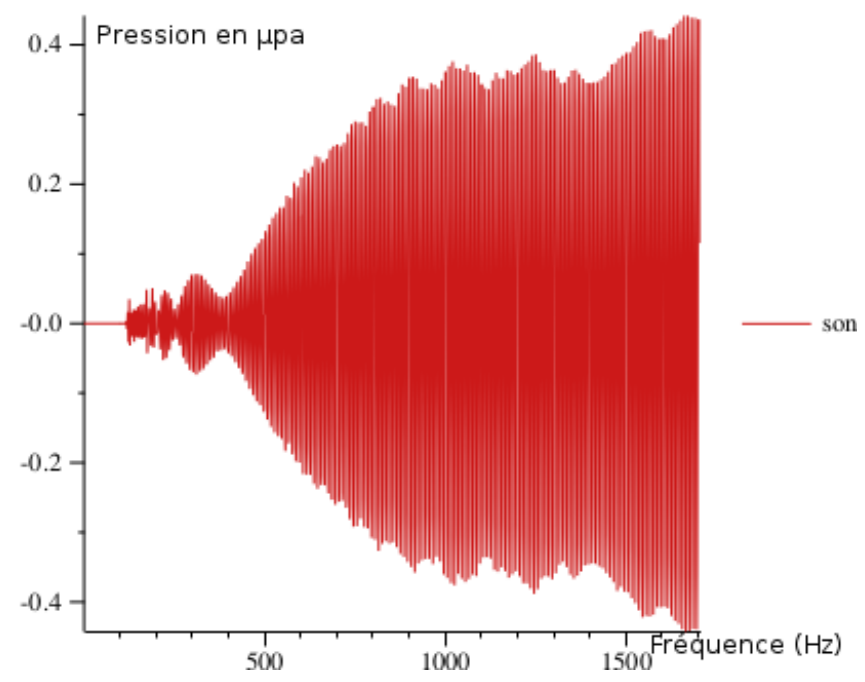

Figure 13. Cell contents of $10 \mathrm{KHz}, 20 \hat{A} t ̦ \mathrm{sec}$

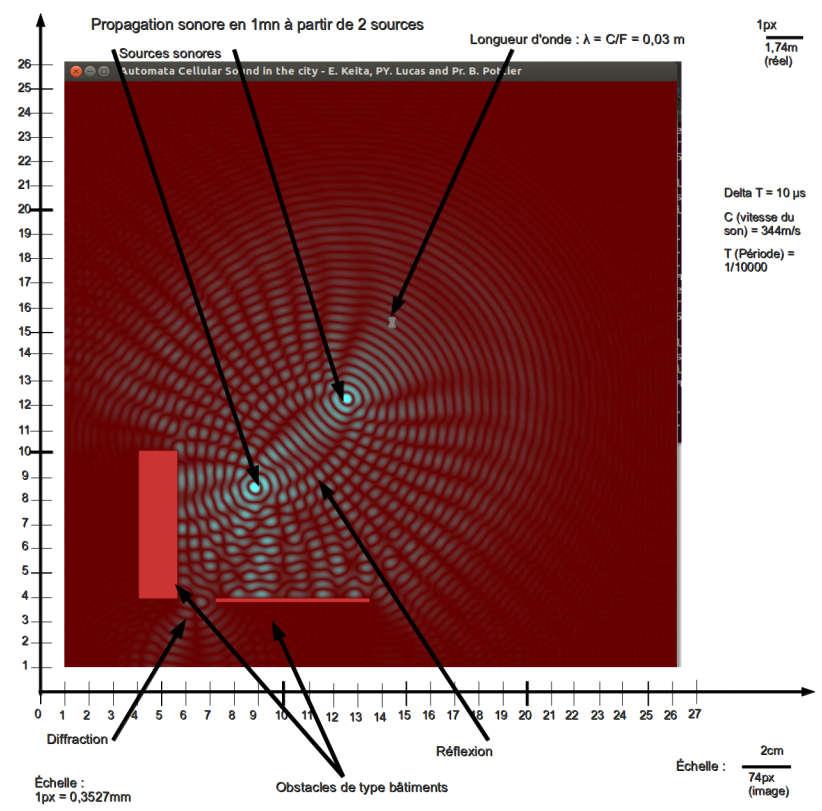

Figure 14. Manual calculation of scale and granularity ...

zone by the sensors. What we have done here is an investigation of the simulation results.

It is interesting in the first place to consider how an actual establishment of the sensors would enable the capture of sound produce by the sound sources. Each sensor provides physical coverage which is its ability to record the neighbouring sound. Figure 16 Presents the degree of coverage of a sensor network according to their number..

Figure 17 presents a statistic for the number of cells in relation to pressure.

One is able to connect the sensors next to several sound sources and to measure therein the noise levels through a 


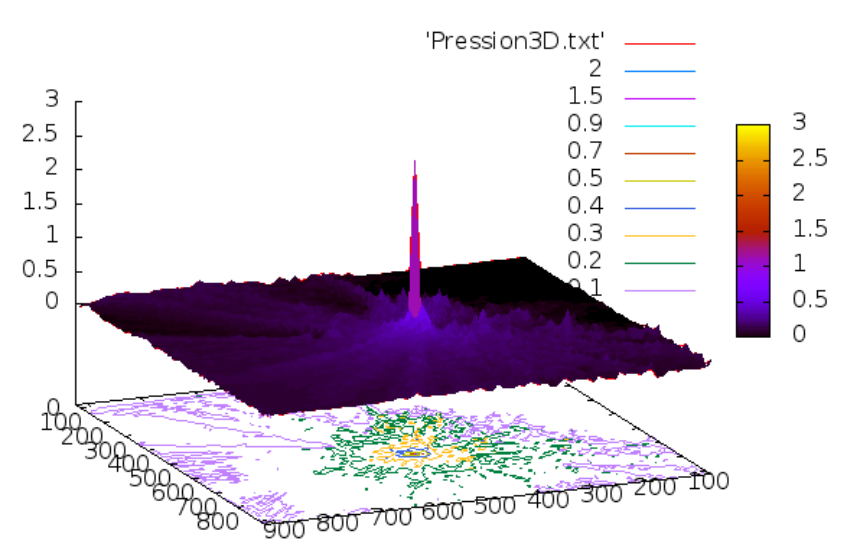

Figure 15. 3D Representation of the pressure in the cell

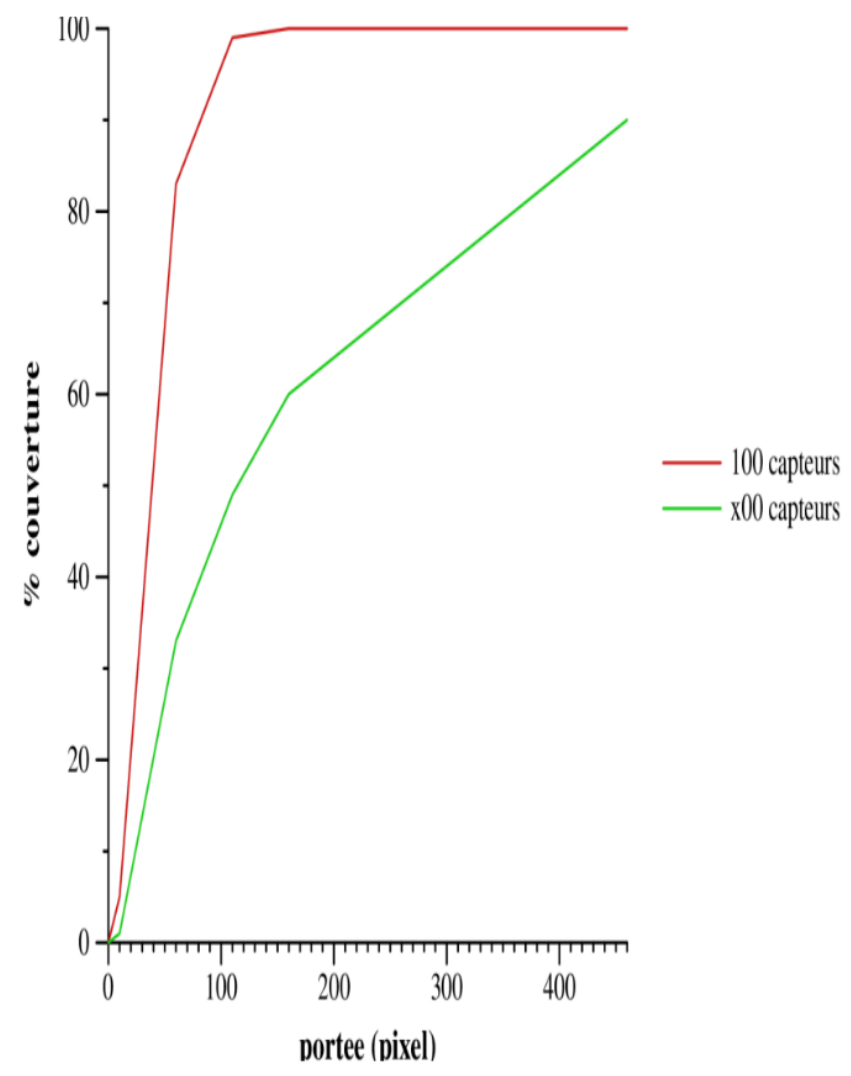

Figure 16. Network coverage, number of sensors concerned

sensor placed at the point representing the sound source, figure:18.

\section{Performance of sequential and parallel executions}

We want to have an idea about the performance and time (duration) of execution in various situations: thus one makes a comparison in terms of execution of speed,

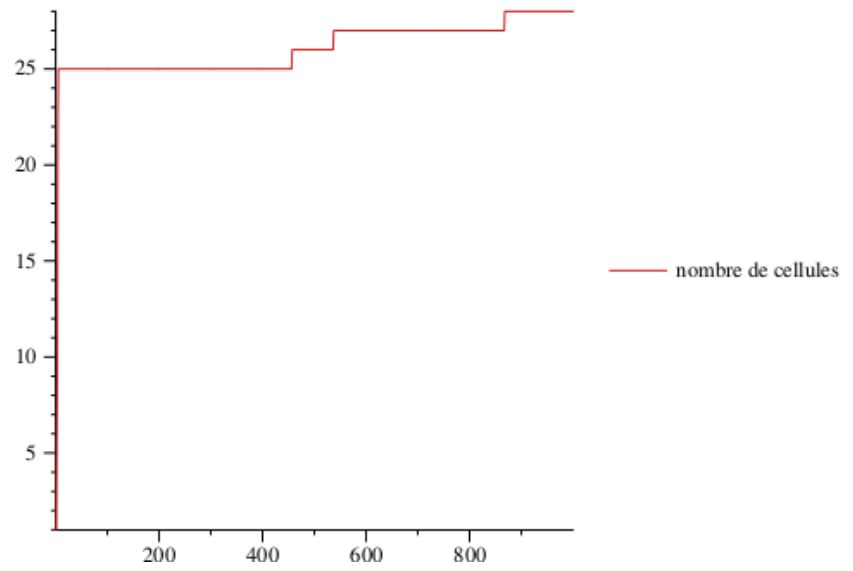

Figure 17. Distribution of the number of cells that have a pressure superior to $1 \mathrm{pa}$ tp $10000 \mathrm{~Hz} \ldots$

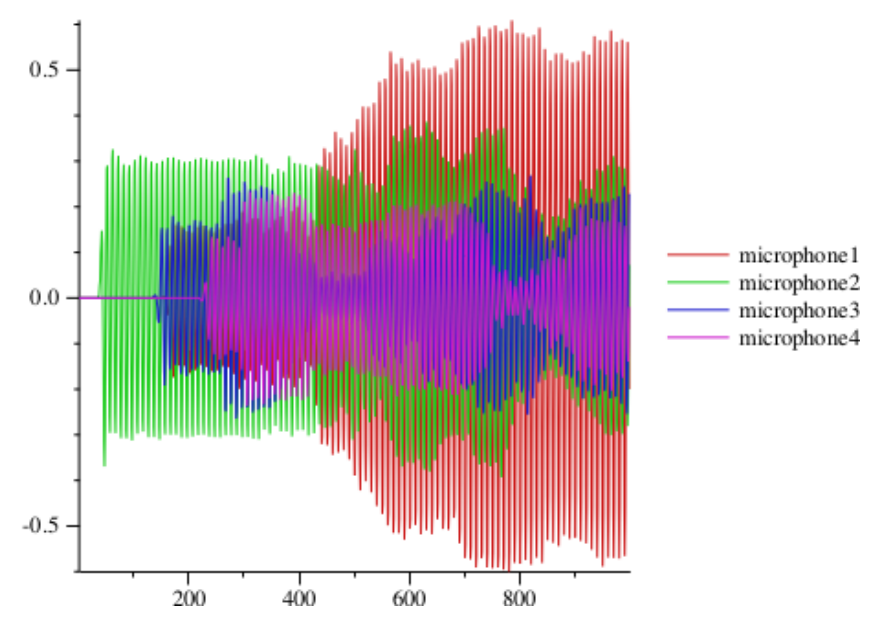

Figure 18. Graphic of the sound level from the sensors placed at the sound source

by varying the number of iterations, the size of the grid cell or still yet, the frequency, etc .. between sequential execution (CPU) and parallel execution ( GPU), figure: 19.

\section{Conclusion and future works}

Cellular automata are used as a discrete approximation of physical phenomena. By choosing the right time scale and good spatial grain, it is possible to represent a situation and its progress in a realistic manner. We applied this execution model to the propagation of sound in an urban context, showing how one or more sound sources may be controlled for transmitting signals that can be received and analyzed after propagation. The application value of this simulation approach is situated in the diagnosis of events, or in situations that affect social life. The parallel execution model is perfectly well suited to the execution of these simulations, and with a clear outcome, the GPU graphic accelerators. The composition of several simulations and their coordination 
- ability to locate satellite sound sources. This would considerably reduce the time taken to pay for an event signaled by a sound source.

\section{References}

[1] J. Von Neumann, A. W. Burks et al., "Theory of selfreproducing automata," IEEE Transactions on Neural Networks, vol. 5, no. 1, pp. 3-14, 1966.

[2] H. Vangheluwe and G. C. Vansteenkiste, "The cellular automata formalism and its relationship to devs." in ESM, 2000, pp. 800-810.

[3] S. A. Gelfand, Hearing: An Introduction to Psychological and Physiological Acoustics. informa Fifth Ed., 2010.

[4] D. G. et son équipe (repris par Gerry Vassilatos dans 'LOST SCIENCE'), "Infrasons (acoustiqua- 1966) - le son silencieux qui tue," NEXUS Num 10, vol. vol.17, janvier 1968.

[5] J. Blauert, Spatial Hearing: the psychophysics of human sound localization. MIT Press, Cambridge, MA Revised Ed., 1983.
[6] C. A. et Eugène de Montalembert, Guide de la théorie de la musique - 610 p. Paris, Fayard (ISBN 978-2-21360977-5), 2001.

[7] R. Dogaru and I. Dogaru., "An efficient sound propagation software simulator based on cellular automata." ISEEE - 3rd International Symposium, Sept. 2010.

[8] M. Vacher and al., "Life sounds extraction and classification in noisy environment." SIP, 2003.

[9] Cartelie, "http://cartelie.application.developpementdurable.gouv.fr/cartelie/

voir.do?carte=d29_carte_de_bruit_a1_2e\&service=ddtm_29\#," en ligne; Page disponible le 7-mars-2015.

[10] Y. I. Toshihiko Komatsuzaki1 and S. Morishita2., "Modelling of incident sound wave propagation around sound barriers using cellular automata," Institute of Science and Engineering, Kanazawa University, Kakuma-machi, Kanazawa, Ishikawa, 920-1192 Japan, 2012.

[11] T. V. Hoang, "Cyber physical systems and mixed simulation," M2RI report, UBO, Tech. Rep., June 2015. 